\title{
研究经尿道电切术治疗非肌层浸润性膀胱癌的效果、 复发情况
}

\author{
张师红* 贾康乐 李炳义 \\ 渭南市第二医院，陕西 714000
}

摘 要: 目的: 观察经尿道电切术在非肌层浸润性膀胱癌临床治疗中的应用价值以及患者的术后复发情况。方 法: 参照组采取常规开放性膀胱部分切除术, 观察组采取经尿道电切术。结果: 与参照组相比较, 观察组的不良反 应发生率、术后复发率均明显下降 $(P<0.05)$, 观察组患者的术中出血量明显下降, 手术操作时长、导尿管留置时 长、住院治疗时长均明显缩短 $(P<0.05)$ 。结论: 经尿道电切术在非肌层浸润性膀胱癌临床治疗中的应用价值较为 理想，可有效缩减手术治疗的处理时间，提升手术治疗的安全性，降低患者的术后复发率，改善患者预后。

关键词：经尿道电切术；非肌层浸润性膀胱癌；临床疗效；复发

\section{一、前言}

非肌层浸润性膀胱癌 (non muscle-invasive bladder cancer) 是一种临床上较为常见的膀胱恶性肿瘤, 在初发膀胱 肿瘤中的占比超过 $70 \%$ 。非肌层浸润性膀胱癌具备易复发、多发性的特征, 极易向深部进展, 因此, 应及时通过手 术方式进行切除, 以防病情进一步恶化。常规开放性膀胱部分切除术、经尿道电切术均是治疗非肌层浸润性膀胱癌的 常用术式 ${ }^{[2]}$ 。本次研究选取2018年1月至2019年12月在我院接受手术治疗的非肌层浸润性膀胱癌患者100例作为研究样 本，着重观察经尿道电切术在非肌层浸润性膀胱癌临床治疗中的应用价值以及患者的术后复发情况，现报告如下。

\section{二、资料与方法}

(一) 一般资料

选取2018年1月至2019年12月, 在我院接受手术治疗的非肌层浸润性膀胱癌患者100例作为研究样本, 将全部人选 患者通过数字随机表划分为两组。参照组病例共计 50 例, 男女比例为 $26: 24$, 年龄中位数为 $(66.43 \pm 2.15)$ 岁, 肿瘤 分期: I期 20 例, II期 30 例; 观察组病例共计 50例, 男女比例为 $27: 23$, 年龄中位数为 $(65.13 \pm 2.22)$ 岁, 肿瘤分期: I 期18例, II期32例。

纳人标准: 均经膀胱镜、CT、超声检查确诊为非肌层浸润性膀胱癌; 意识清醒; 受教育程度为小学及以上; 择期 进行手术治疗。

排除标准: 伴随严重的肝肾等脏器器官功能障碍; 合并严重的心脑血管疾病; 伴随认知障碍或精神类疾病; 伴随 凝血功能障碍；合并恶性肿瘤或免疫系统疾病。

\section{(二) 方法}

参照组采取常规开放性膀胱部分切除术。患者保持仰卧体位, 术前留置导尿管, 手术切口选取患者耻骨上方正中 位置处, 切开膀胱, 充分暴露肿瘤。围绕肿瘤中心膀胱壁将肿瘤周边 $2 \sim 3 \mathrm{~cm}$ 的正常膀胱壁切除, 同时需要对粘连腹膜 实施切除处理。若肿瘤位置与输尿管口相接近, 需同时将输尿管口、输尿管下段切除, 并对输尿管残端进行移植, 将 其移植至无肿瘤的膀胱壁上。术后留置双 $\mathrm{J}$ 管。观察组采取经尿道电切术。予以患者腰麻或全麻处理, 将电切镜的电 切功率设定为 $120 \sim 160 \mathrm{~W}$, 电凝功率设定为 $60 \sim 80 \mathrm{~W}$, 以等渗液作为冲洗液。予以患者常规消毒铺巾处理, 经尿道置 人电切镜, 对肿瘤的位置、大小、数目以及其与输尿管的间距进行仔细观察 ${ }^{[3]}$ 。对于体积较小的肿瘤, 应同时切除肿 瘤及基底部膀胱壁, 注意必须切除至肌层; 对于体积较大的肿瘤, 应先将膀胼壁表面明显突出的肿物逐步切除, 待其 彻底清除后再对基底部肿物进行切除, 注意必须切除至肌层, 最后将肿瘤周边 $2 \sim 3 \mathrm{~cm}$ 的正常膀胱壁切除。若肿瘤位置 与输尿管口相接近, 必须迅速切除, 尽量避免应用电凝切割。对于多发性肿瘤, 切除操作必须遵循 “先易后难、先小

*通讯作者: 张师红, 1976年12月, 男, 汉族, 陕西渭南人, 现任职于渭南市第二医院泌尿外科, 副主任医师, 本科。研究方向; 经皮肾铁激光碎石及腹腔镜下泌尿系肿瘤的根治。 
后大、侧壁肿瘤最后切除”的基本原则。手术完成后需留置导尿管，反复冲洗膀胱直至干净。

(三) 观察指标

1. 对两组患者术中出血量、手术操作时长、导尿管留置时长、住院治疗时长进行对比分析。

2. 对两组患者不良反应的发生情况进行对比分析。不良反应：闭孔神经反射、膀胱穿孔、膀胱痉挛。

3. 对两组患者的术后复发情况进行对比分析。

(四) 统计学处理

统计分析软件选用SPSS 20.0 , 计量资料采用 $t$ 检验, 结果数据的表现形式为 $\bar{x} \pm s$; 计数资料采用 $\chi^{2}$ 检验, 结果数 据的表现形式为率。 $P<0.05$ 认定差异存在统计学意义。

\section{三、结果}

（一）两组患者术中出血量、手术操作时长、导尿管留置时长、住院治疗时长对比

对两组患者的术中出血量、手术操作时长、导尿管留置时长、住院治疗时长进行组间对比, 差异存在统计学意义 $(P<0.05)$ 。与参照组相比较, 观察组患者的术中出血量明显下降, 手术操作时长、导尿管留置时长、住院治疗时 长均明显缩短 $(P<0.05)$ 。如表1所示。

表1 两组患者术中出血量、手术操作时长、导尿管留置时长、住院治疗时长对比

\begin{tabular}{ccccc}
\hline 组别 & 术中出血量 $(\mathrm{mL})$ & 手术操作时长 $(\mathrm{min})$ & 导管留置时长 $(\mathrm{d})$ & 住院治疗时长 $(\mathrm{d})$ \\
\hline 参照组 $(n=50)$ & $99.32 \pm 12.32$ & $98.25 \pm 18.25$ & $11.33 \pm 1.34$ & $15.45 \pm 2.01$ \\
观察组 $(n=50)$ & $42.43 \pm 11.94$ & $41.73 \pm 15.37$ & $5.23 \pm 1.19$ & $7.02 \pm 1.78$ \\
$\chi^{2}$ & 8.053 & 9.494 & 8.288 & 7.286 \\
$P$ & 0.001 & 0.001 & 0.001 & 0.001 \\
\hline
\end{tabular}

（二）两组患者不良反应的发生情况对比

参照组与观察组患者的不良反应发生率分别为 $20.00 \% 、 4.00 \%$ 。对两组患者的不良反应发生率进行组间对比, 差异 存在统计学意义 $(P<0.05)$ 。与参照组相比较，观察组患者的不良反应发生率明显下降 $(P<0.05)$ 。如表2所示。

表2 两组患者不良反应的发生情况对比[例数（\%)]

\begin{tabular}{|c|c|c|c|c|}
\hline 组别 & 闭孔神经反射 & 膀胱穿孔 & 膀胱痉挛 & 发生率 \\
\hline 参照组 $(n=50)$ & $3(6.00)$ & $2(4.00)$ & $5(10.00)$ & $10(20.00)$ \\
\hline 观察组 $(n=50)$ & $0(0.00)$ & $1(2.00)$ & $1(2.00)$ & $2(4.00)$ \\
\hline$\chi^{2}$ & & & & 7.494 \\
\hline$P$ & & & & 0.002 \\
\hline
\end{tabular}

（三）两组患者的术后复发情况对比

参照组 50 例患者中有 9 例术后复发患者, 即术后复发率为 $18.00 \%$, 观察组 50 例患者中有 2 例术后复发患者, 即术后 复发率为 $4.00 \%$ 。对两组患者的术后复发率进行组间对比, 差异存在统计学意义 $(P<0.05)$ 。与参照组相比较, 观察 组患者的术后复发率明显下降 $(P<0.05)$ 。

\section{四、讨论}

肌层浸润性膀脱癌从肿瘤生长的生物学特性或者恶性度方面, 膀胱癌具备易复发、多发性的特征, 通常需要多次 重复手术。膀胱癌 $\mathrm{T}_{\mathrm{a}}$ 期和 $\mathrm{T}_{1}$ 期虽然都属于非肌层浸润性膀胱癌, 但二者的生物学特性有显著不同。由于固有层内血管 和淋巴管丰富, 因此 $\mathrm{T}_{1}$ 期的膀胱癌更容易发生肿瘤扩散。相关研究指出, 膀胱癌术后并发症主要为闭孔神经反射、膀 胱穿孔、膀胱痉挛, 其中以膀胱穿孔的发生概率为最高, 由于腹膜在膀胱顶部与后壁均有覆盖, 故而极易发生腹膜穿 孔; 闭孔神经反射的诱发机制主要为术中电流极易穿过膀胱壁从而对闭孔神经产生一定的刺激作用, 致使大腿内收肌 群收缩、骨盆移动。常规开放性膀胱部分切除术、经尿道电切术均是治疗非肌层浸润性膀胱癌的常用术式 ${ }^{[4]}$ 。但常规 开放性膀胱部分切除术极易对患者造成较大的创伤, 术中出血量比较大, 而且术后极易复发, 术后并发症的发生风 险相对较高, 致使患者的术后康复进程延缓。经尿道电切术是一种微创手术疗法, 该术式将在患者的膀胱内开展手术 操作模式, 因此, 不会给患者造成太大的创伤, 同时有助于阻止肿瘤组织的进一步扩散, 而且不会对患者的膀胱功能 产生任何不良影响。应用经尿道电切术切除病灶时, 可同时进行止血操作, 这在一定程度上减缓了患者的术中出血情 
况, 降低了患者的术中出血量 ${ }^{[5]}$ 。此外, 经尿道电切术中不存在组织凝块, 手术视野较为清晰, 有利于缩短手术操作 时长, 从而降低闭孔神经反射、膀胱穿孔、膀胱痉挛等不良反应的发生率 ${ }^{[6-7]}$ 。相关研究证实, 经尿道电切术较开放性 膀胱部分切除术并发症更少, 患者的耐受性更佳, 安全性更高, 术后较短时间内便可取出留置的导尿管, 有利于加快 患者病情的康复进程。本次研究发现, 与参照组相比较, 观察组患者的术中出血量明显下降, 手术操作时长、导尿管 留置时长、住院治疗时长均明显缩短; 参照组与观察组患者的不良反应发生率分别为 $20.00 \% 、 4.00 \%$, 观察组患者的 不良反应发生率明显低于参照组; 参照组与观察组患者的术后复发率分别为 $18.00 \% 、 4.00 \%$, 观察组患者的术后复发 率明显低于参照组。提示相比于常规开放性膀胱部分切除术, 经尿道电切术可有效改善非肌层浸润性膀胱癌患者的临 床症状，加快患者的术后康复进程，有利于降低闭孔神经反射、膀胱穿孔、膀胱痉挛等不良反应的发生率以及术后复 发率，从而提升手术治疗的安全性。

\section{五、结论}

综上所述，经尿道电切术在非肌层浸润性膀胱癌临床治疗中的应用价值较为理想，可有效缩减手术治疗的处理时 间，提升手术治疗的安全性，降低患者的术后复发率，改善患者预后。

\section{参考文献:}

[1]马进华, 魏红兵, 李先林, 潘峰, 陈德红. 经尿道钬激光切除术对非肌层浸润性膀胱癌患者氧化应激因子和肿瘤相关 因子的影响 [J].新疆医科大学学报, 2020,43(02):158-162.

[2]康慎, 聂仕正. 非肌层浸润性膀胱癌治疗中经尿道膀胱肿瘤电切术与经尿道膀胱肿瘤整块切除术的效果分析 [J]. 山 西医药杂志, 2020,49(03):247-249.

[3]薛炜,邱建新,桑楠,张志明.影响非肌层浸润性膀胱癌患者经尿道膀胱肿瘤电切术后复发的相关因素分析 [J]. 实用 医院临床杂志, 2019,16(05):30-33.

[4]李亚伟, 程浪, 黄后宝. 经尿道膀胱肿瘤海博刀整块切除与经尿道膀胱肿瘤电切治疗非肌层浸润性膀胱癌的疗效及 安全性对比分析[J].现代泌尿生殖肿瘤杂志, 2019,11(04):209-215.

[5]费安华, 付明,胡刚强, 万建. 经尿道膀胱肿瘤电切术治疗高级别非肌层浸润性膀胱癌的疗效分析 [J]. 癌症进展, 2019,17(15):1839-1842.

[6]邹义华, 李勇, 张威, 陈晓峰, 陈善群. $1470 \mathrm{~nm}$ 激光经尿道膀胱肿瘤整块切除术治疗非肌层浸润性膀胱癌的疗效观察 (附55例报告)[J].中国内镜杂志, 2019,25(12):80-83.

[7]李中华.经尿道膀胱癌等离子电切术联合吉西他滨治疗高龄高危非肌层浸润性膀胱癌患者的临床疗效探析 [J]. 当 代医学, 2019,25(20):138-139. 